\title{
Orbital tuning, eccentricity, and the frequency modulation of climatic precession
}

\author{
Peter Huybers $^{1}$ and Oded Aharonson ${ }^{2}$ \\ Received 23 February 2010; revised 10 September 2010; accepted 23 September 2010; published 23 December 2010.
}

[1] The accuracy of geologic chronologies can, in principle, be improved through orbital tuning, the systematic adjustment of a chronology to bring the associated record into greater alignment with an orbitally derived signal. It would be useful to have a general test for the success of orbital tuning, and one proposal has been that eccentricity ought to covary with the amplitude envelope associated with precession variability recorded in tuned geologic records. A common procedure is to filter a tuned geologic record so as to pass precession period variability and compare the amplitude modulation of the resulting signal against eccentricity. There is a reasonable expectation for such a relationship to be found in paleoclimate records because the amplitude of precession forcing depends upon eccentricity. However, there also exists a relationship between eccentricity and the frequency of precession such that orbital tuning generates eccentricity-like amplitude modulation in filtered signals, regardless of the accuracy of the chronology or the actual presence of precession. This relationship results from the celestial mechanics governing eccentricity and precession and from the interaction between frequency modulation and amplitude modulation caused by filtering. When the eccentricity of Earth's orbit is small, the frequency of climatic precession undergoes large variations and less precession energy is passed through a narrow-band filter. Furthermore, eccentricity-like amplitude modulation is routinely obtained from pure noise records that are orbitally tuned to precession and then filtered. We conclude that the presence of eccentricity-like amplitude modulation in precession-filtered records does not support the accuracy of orbitally tuned time scales.

Citation: Huybers, P., and O. Aharonson (2010), Orbital tuning, eccentricity, and the frequency modulation of climatic precession, Paleoceanography, 25, PA4228, doi:10.1029/2010PA001952.

\section{Introduction}

[2] Earth's orbital configuration can be calculated to a high degree of accuracy over the past tens of millions of years [Laskar et al., 2004]. Therefore, orbital variations offer the possibility of demarking the flow of time in geologic records if their signals can be continuously tracked. This possibility has long been recognized [McGee, 1892; Gilbert, 1900], but only with the unambiguous identification of orbital period variability in marine sediment core records [Hays et al., 1976] did orbital tuning become a standard practice [e.g., Imbrie et al., 1984; Shackleton et al., 1990; Lisiecki and Raymo, 2005]. The general approach is to stretch, squeeze, and shift portions of a climate record so as to maximize its correspondence with a curve derived from the time history of changes in Earth's orbital configuration, a process referred to as orbital tuning. Note that changes in insolation result from both orbital (e.g., eccentricity) and

\footnotetext{
${ }^{1}$ Department of Earth and Planetary Sciences, Harvard University, Cambridge, Massachusetts, USA.

${ }^{2}$ Division of Geological and Planetary Sciences, California Institute of Technology, Pasadena, California, USA.

Copyright 2010 by the American Geophysical Union. 0883-8305/10/2010PA001952
}

rotational (e.g., precession and changes in the obliquity of Earth's spin axis) changes but that we use orbital to refer to all changes in Earth's orbit and orientation that result in long-term changes in the distribution of insolation.

[3] Several distinct methods exist to check the accuracy of orbitally tuned records. One well-known success was the prediction of an older date for the Brunhes-Matuyama magnetic reversal than had been estimated using radiometric methods [Johnson, 1982; Shackleton et al., 1990] and which was subsequently confirmed with more accurate radiometric estimates. More generally, independently determined dates provide checks on orbital tuning's results, but these are usually only available at finite horizons and only convincing when fully withheld from the tuning process prior to comparison. A second test involves tuning to a single orbital band, e.g., that associated with precession, and then evaluating success using the concentration of variance at other bands, e.g., obliquity [Hilgen et al., 1993; Karner et al., 2002]. This minimal tuning approach is generally applicable but requires about half the orbital signal be reserved for testing. An additional check upon the accuracy of an orbital time scale can be obtained by tuning distinct climate records, for example, as was done for the marine $\delta^{18} \mathrm{O}$ record by tuning Mediterranean sapropel records [Lourens et al., 1996; Lourens, 2004], though the stringency of such a check depends upon the degree to which the tuned signals are 
independent of one another and the accuracy with which the resulting time scales can be related to one another.

[4] A final test, which is the focus of this study, involves comparing eccentricity against the amplitude modulation of variability in the precession band of a tuned record [e.g., Imbrie et al., 1984; Ruddiman et al., 1989; Shackleton et al., 1990; Tiedemann et al., 1994; Shackleton et al., 1995; Paillard, 2001]. We illustrate this test using the planktic $\delta^{18} \mathrm{O}$ record from Ocean Drilling Program ODP 677 [Shackleton et al., 1990] (ODP 677) it is relatively long and well-resolved, and because Shackleton et al. [1990] obtained a good correlation between eccentricity and the amplitude of the precession variability in this record. Specifically, we narrow-passband filter the ODP 677 record using a fourthorder Butterworth filter and then take the Hilbert transform to estimate the amplitude envelope of the resulting signal [e. g., Bracewell, 2000]. A question arises as to what frequencies should be passed by the filter, and a search is made of high-frequency cutoffs ranging between $1 / 14$ and $1 / 20$ $\mathrm{kyr}^{-1}$ and low-frequency cutoffs between $1 / 21$ and $1 / 27$ $\mathrm{kyr}^{-1}$. Passing of frequencies between $1 / 18 \mathrm{kyr}^{-1}$ and $1 / 24$ $\mathrm{kyr}^{-1}$ is found to maximize the cross correlation of the resulting amplitude envelope with eccentricity, giving a value of 0.61 (see Figure 1).

[5] A point of comparison is available through repeating the same analysis on an untuned version of the ODP 677 $\delta^{18} \mathrm{O}$ record. Time is interpolated with depth between the geomagnetic reversal dates of Berggren et al. [1985], where core depths for each reversal are taken from Shackleton et al. [1990]. The standard deviation between the resulting depth-derived and orbitally tuned time estimates is $40 \mathrm{kyr}$. Repeating the same filtering search described above yields a maximum cross correlation of less than 0.12. Thus, a markedly higher correlation is obtained when the $\delta^{18} \mathrm{O}$ record is placed upon the tuned time scale.

[6] The appearance of such eccentricity-like amplitude modulation in filtered paleoclimate records has been cited as lending strong support for the existence of orbital forcing within the climate system, as well as for corroborating the accuracy of paleoclimate time scales. For example, Imbrie et al. [1984, p. 269] stated that the "statistical evidence of a close relationship between the time-varying amplitudes of orbital forcing and the time-varying amplitudes of the isotopic response implies that orbital variations are the main external cause of the succession of late Pleistocene ice ages." Shackleton et al. [1990, p. 257] stated that "[t]he resemblance between the eccentricity in the model output and the modulation on the filtered planktonic data is remarkable, and it seems very unlikely that this match could have been obtained with an incorrect timescale." Shackleton et al. [1995, p. 696] concluded that, "[p]robably the most important feature through which the orbital imprint may be unambiguously recognized in ancient geological records is the amplitude modulation of the precession component by the varying eccentricity of the Earth orbit." As a final example, in comparing a tuned and narrow-band-pass filtered record against precession, Paillard [2001, p. 329] stated that "[i]t is remarkable that both time series have a quite similar modulation of their amplitude. This is probably one of the strongest arguments in favor of a simple causal relationship between the precessional forcing and the climatic response in this frequency band. Indeed, in contrast to other techniques, amplitude modulation is not affected by tuning."

[7] But there has been some criticism of this eccentricity amplitude modulation test. In a technical report, Neeman [1993] presented evidence that orbital tuning influences amplitude modulation. His approach was to tune synthetic noise signals and demonstrate that, after filtering, eccentricity-like amplitude modulation appeared in the precession band variability. This result was also discussed in the book by Muller and MacDonald [2000] and reproduced by Huybers and Wunsch [2004, Appendix C]. However, a mechanistic explanation for the appearance of eccentricitylike amplitude modulation has been lacking. Here we seek to explain the origin of eccentricity-like amplitude modulation in tuned records using concepts drawn from celestial mechanics and signal processing.

\section{How Eccentricity Influences the Frequency of Precession}

[8] The precession of Earth's spin axis, when measured with respect to inertial space, occurs with a nearly constant $25.7 \mathrm{kyr}$ period - excepting the small and much higherfrequency effects associated with nutation - as a result of Earth's gravitational interaction with the Moon, Sun, and other planets [e.g., Williams, 1994]. But it is the orientation of Earth's spin axis with respect to its eccentric orbit that determines the pattern of incoming solar radiation. The relevant angle, $\tilde{\omega}$, is measured between the moving Northern Hemisphere spring equinox and perihelion along Earth's orbit [e.g., Laskar et al., 1993]. The frequency associated with $\tilde{\omega}$ averages $1 / 22.1 \mathrm{kyr}^{-1}$, as opposed to $1 / 25.7 \mathrm{kyr}^{-1}$, because perihelion tends to move toward spring equinox, though the mean is only a partial description of this irregular movement. For example, the solution of Laskar et al. [2004] indicates that $374 \mathrm{kyr}$ ago $\mathrm{d} \tilde{\omega} / d t$ was $\sim 2 \pi / 13 \mathrm{kyr}^{-1}$ and $536 \mathrm{kyr}$ ago it was $2 \pi / 33 \mathrm{kyr}^{-1}$, even after smoothing the frequency variations using an $11 \mathrm{kyr}$ window. Berger [1976] also noted this irregularity in the precession frequency.

[9] The importance of $\tilde{\omega}$ for insolation depends directly on the eccentricity of Earth's orbit, and a useful term for describing this relation is $e \sin \tilde{\omega}$, referred to as the climatic precession parameter, where $e$ is eccentricity. The $\sin \tilde{\omega}$ term is largest when perihelion aligns with northern hemisphere summer solstice, $90^{\circ}$ of solar longitude after spring equinox. It is worth noting that terms such as the climatic precession parameter always appear in the full representations of insolation forcing as modulation of the annual cycle or its harmonics [e.g., Rubincam 1994]. As has long been recognized [Herschel, 1832], precession influences the timing and amplitude of the annual cycle of insolation but does not change the annual average insolation at any latitude. Thus, some nonlinear response to insolation forcing or nonlinear recording of the response needs to occur for precession terms to appear in the climate record, but given the wide range of physical and recording nonlinearities that are pos- 

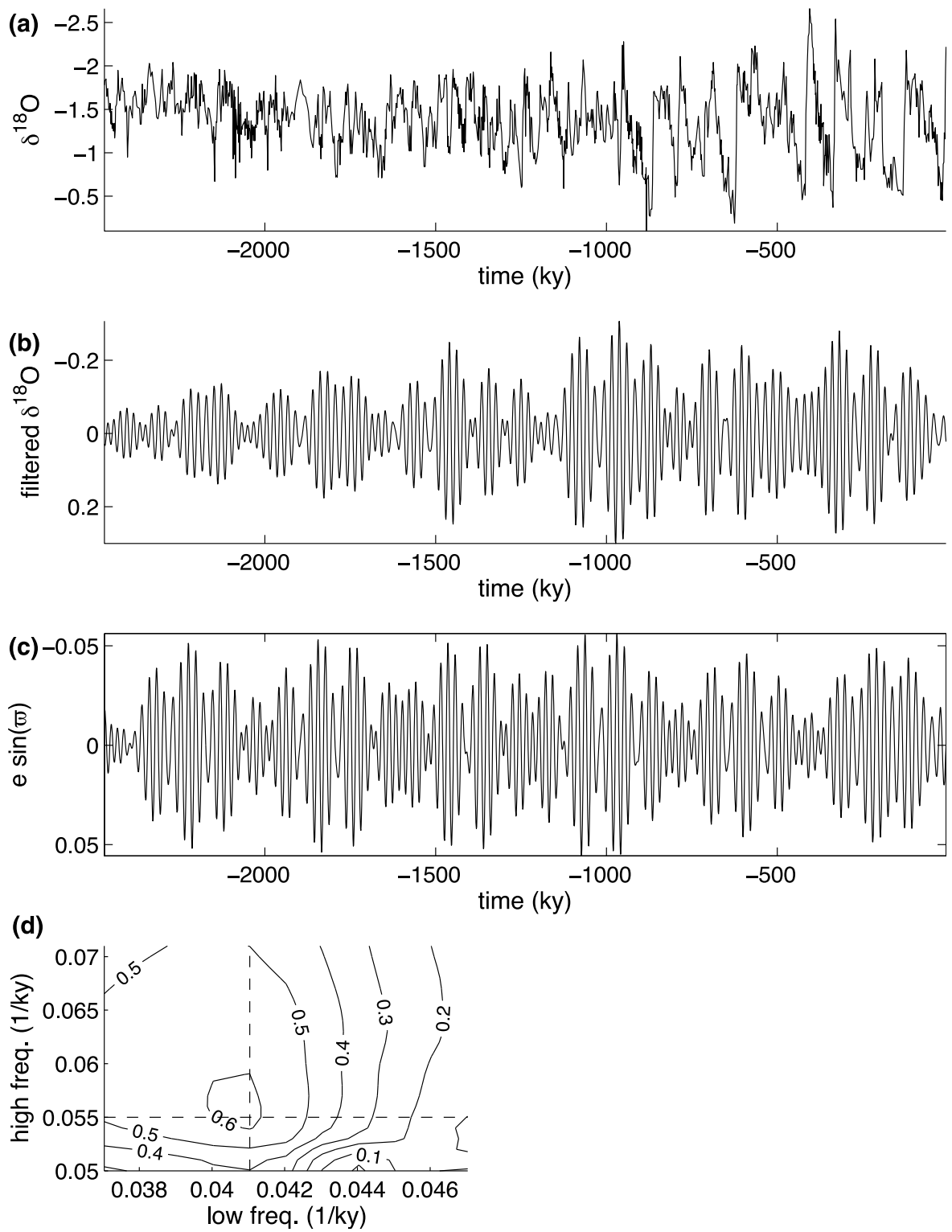

Figure 1. Evoking eccentricity-like amplitude modulation. (a) ODP $677 \delta^{18} \mathrm{O}$ record on an orbitally tuned time scale [Shackleton, 1990]. (b) Filtered version of the orbitally tuned record using cutoff frequencies of $1 / 24$ and $1 / 18 \mathrm{kyr}^{-1}$, chosen to maximize the cross correlation between the amplitude of the resulting precession period variability and eccentricity. (c) Climatic precession [Laskar, 2004], showing an amplitude envelope correlated with Figure 1b. (d) Cross correlation between eccentricity and the envelope of the filtered record using different combinations of high and low cutoff frequencies, giving a maximum of 0.61 . A similar analysis using a non-orbitally tuned version of the ODP $677 \delta^{18} \mathrm{O}$ record yields a maximum cross correlation of no more than 0.12. Note that Shackleton [1990] used an orbital solution from Berger [1989], whereas we use the more recent solution of Laskar [2004], and that these yield consistent results.

sible [e.g., Huybers and Wunsch, 2004], it is not surprising to find precession signals in paleoclimate records.

\subsection{The Frequency of Climatic Precession}

[10] The influence of eccentricity on the amplitude of precession forcing is more widely appreciated than its influence upon the frequency. The degree to which a grav- itational perturbation influences Earth's orbital parameters depends on the strength and orientation of the perturbing force, as well as Earth's orbital configuration itself. Burns [1976] showed that the change in $\tilde{\omega}$ resulting from a gravitational perturbation will be proportional to $e^{-1}\left(1-e^{2}\right)^{1 / 2}$, suggesting that during times of low eccentricity $d \tilde{\omega} / d t$ will have greater variability. 
[11] The foregoing simple example approximates the perturbations to Earth's orbit as instantaneous pulses, but in actuality prolonged exchanges of momentum occur between Earth and other celestial bodies. These interactions can be better described using secular theory, where perturbations to the planets are approximated by interacting elliptical rings of mass distributed along their orbits [e.g., Murray and Dermott, 1999]. Appendix A describes the relationship between eccentricity and the frequency of precession using secular theory for the case of a single orbit perturbed by one other orbiting mass. This depiction, albeit simple, captures the primary features observed in more complete orbital solutions and demonstrates the link between small eccentricity and large anomalies in the precession frequency. The secular solution could be expanded to approximate the evolution of the solar system, but it is simpler to appeal directly to a numerical simulation.

\subsection{Analysis of Laskar's Solution}

[12] The history of Earth's orbital variations is available from analytical [Laskar, 1988], semi-analytical [Laskar et al., 1993], and numerical integration [Quinn et al., 1991; Laskar et al., 2004]. Beyond tens of millions of years ago, the chaotic nature of Earth's orbit precludes accurate estimation of its orbital state [Laskar et al., 2004], but over the past few million years there is less difficulty [Lourens et al., 2004]. Changes in Earth's mass distribution and tidal coupling with the Moon could also significantly influence the orbital solution [Laskar et al., 1993, 2004], even over the past five $\mathrm{Ma}$, but these additional considerations are not treated here. The aforementioned limitations in predicting the exact orbital configuration are not expected to affect the fundamental interactions between eccentricity and precession.

[13] The frequency of precession over the past 5 million years is computed by differencing numerical estimates of $\tilde{\omega}$ at $1 \mathrm{kyr}$ intervals [Laskar et al., 2004]. As expected, deviations in the frequency grow with decreasing eccentricity (Figure 2). When Earth's eccentricity is below 0.01, only $44 \%$ of the estimated frequencies reside between $1 / 18$ and $1 / 24 \mathrm{kyr}^{-1}$, the band choice that maximized the correlation for the results derived from the ODP 677 record (Figure 1). Similar results hold for any interval of Laskar's [2004] orbital solution, ranging from $50 \mathrm{Myr}$ before the present to $20 \mathrm{Myr}$ after the present. During times of low eccentricity, the instantaneous frequency associated with precession tends to stray outside the typical bounds used to define the precession band. This suggests that filtering records tuned to precession could have a systematic influence upon the amplitude structure of the resulting precession variability.

\section{Connection Between Frequency and Amplitude Modulation in Filtered Signals}

[14] Given that climate variability occurs at all time scales, filtering is a natural means of isolating precession variability in paleoclimate records, but it can have consequences for the amplitude of the resulting signal. Apparently, if the filter is centered on the mean precession frequency, the resulting signal will tend to have a lower amplitude when the instantaneous frequency strays outside the filter's bandwidth.

\subsection{Simple Example}

[15] For purposes of illustration, consider a sinusoid whose frequency is modulated by another sinusoid, $x(t)=$ $\sin \left(2 \pi f_{c} t+\beta \sin \left(2 \pi f_{m} t\right)\right)$, where $f_{c}$ is the carrier frequency and $f_{m}$ is the frequency of the modulation. The amplitude of the modulation is given by $\beta$. This frequency-modulated signal can also be expressed as a summation of individual sinusoids [e.g., Olver, 1962, equations 9.1.44-45]

$$
\begin{aligned}
x(t)= & J_{(0, \beta)} \sin \left(2 \pi f_{c} t\right)+\sum_{k=1}^{\infty} J_{(k, \beta)}(-1)^{k}\left[\sin \left(2 \pi\left(f_{c}-k f_{m}\right) t\right)\right. \\
& \left.+(-1)^{k} \sin \left(2 \pi\left(f_{c}+k f_{m}\right) t\right)\right],
\end{aligned}
$$

where the $J_{k, \beta}$ terms are order $k$ Bessel functions of the first kind, evaluated at $\beta$. The $f_{c} \pm k f_{m}$ terms indicate that fully describing a frequency-modulated signal can require an arbitrarily large bandwidth as $k$ increases. Whereas $x(t)$ has no amplitude modulation, any filtering that removes energy at frequencies $f_{c} \pm k f_{m}$ can be expected to yield a signal with some amplitude modulation.

[16] In the case that all terms with $k>1$ are filtered, equation (1) can be expressed as follows:

$$
\tilde{x}(t)=J_{(0, \beta)} \sin \left(2 \pi f_{c} t\right)-J_{1, \beta} \cos \left(2 \pi t f_{m}\right) \sin \left(2 \pi t f_{c}\right) .
$$

The last term in equation (2) indicates amplitude modulation of a carrier signal with frequency $f_{c}$ by a sinusoid with frequency $f_{m}$. If the size of $J_{1, \beta}$ is non-negligible, $\tilde{x}(t)$ will have an amplitude modulation whose structure is determined by the frequency modulation of $x(t)$.

[17] As the frequency modulation of climatic precession is episodic, as opposed to periodic, it is unclear what amplitude to assign the frequency modulation term, but as an example, if $\beta$ is taken as $0.8 \pi$, the spectrum associated with equation (1) is in reasonable agreement with the spectral estimate of $\sin \tilde{\omega}$, and $J_{1 \beta}$ is many times larger than $J_{0, \beta}$. In this case, filtering turns the purely frequency-modulated signal into a signal with substantial amplitude modulation. Thus, orbital tuning, which will influence frequency modulation, can also influence the amplitude structure of a signal once filtering is involved.

[18] Climatic precession contains a more complicated frequency modulation than the simple example considered above, making it also useful to explore the influence of filtering upon the actual orbital elements. Consider $\sin (\tilde{\omega})$, which is frequency modulated but not amplitude modulated (Figure 3). Applying the same Butterworth filter to $\sin (\tilde{\omega})$ found to be optimal for filtering the ODP $677 \delta^{18} \mathrm{O}$ record yields a signal whose amplitude modulation closely resembles the variations in eccentricity, where these quantities have a cross correlation of 0.86 (Figure 3 ). This result can be understood, first, in that anomalies in the frequency of precession tend to be larger when eccentricity is smaller, and, second, in that filtering tends to reduce the variance of the signal at times of large anomalies in the frequency associated with $\tilde{\omega}$. 

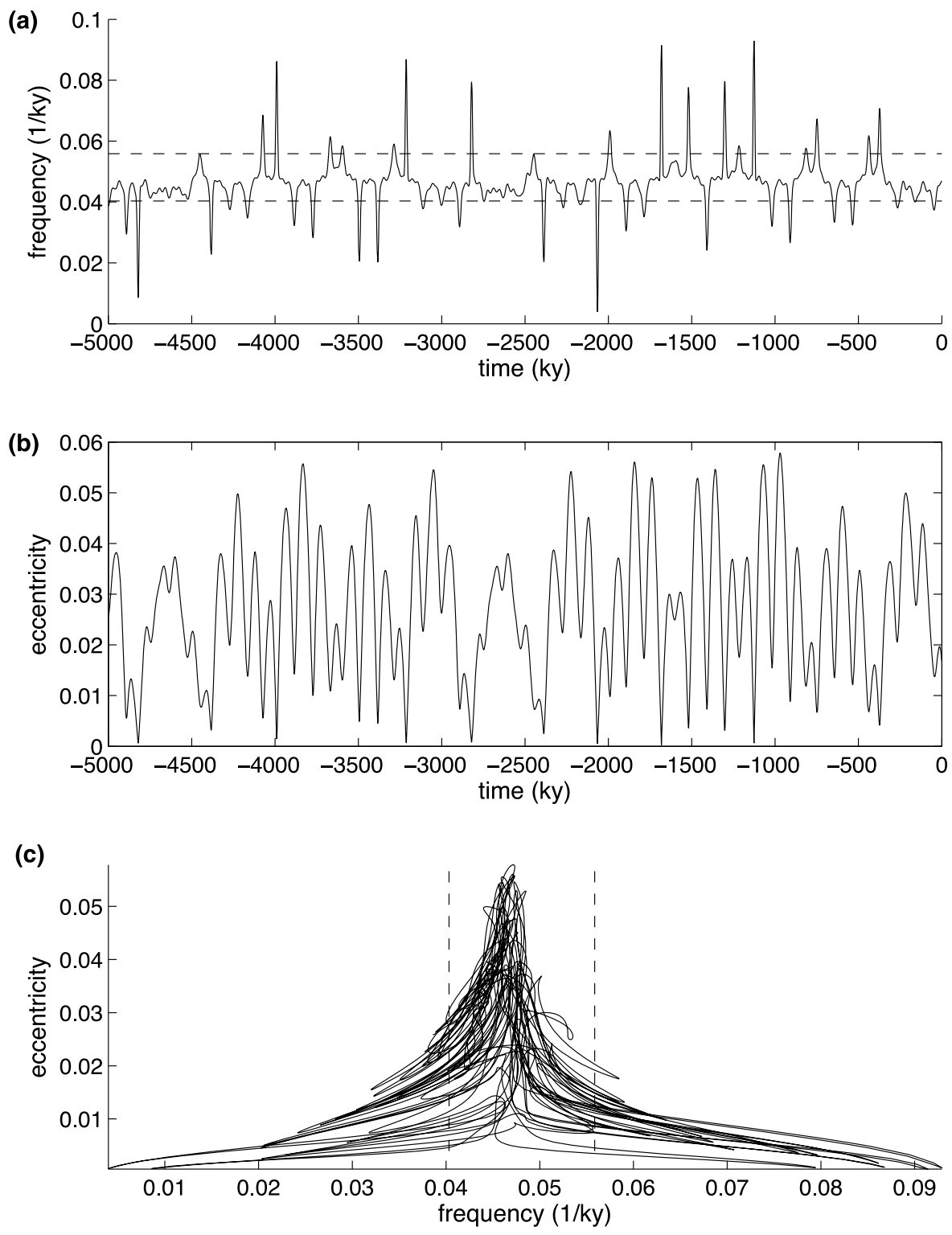

Figure 2. Relationship between precession and eccentricity. (a) Frequency associated with the angle between Northern Hemisphere spring equinox and perihelion, $d \tilde{\omega} / d t$. Dashed lines indicate frequencies of $1 / 18$ and $1 / 24 \mathrm{kyr}^{-1}$ (see Figure 1). (b) Earth's orbital eccentricity. For visual clarity, $d \tilde{\omega} / d t$ was smoothed with an $11 \mathrm{kyr}$ weighted running average prior to plotting. (c) Eccentricity plotted against $d \tilde{\omega} / d t$, illustrating how large excursions in frequency occur during low eccentricity. Dashed lines are at the same frequencies as in Figure 2a. Orbital values are from the solution of Laskar [2004].

\subsection{Synthetic Test}

[19] The eccentricity cross correlation obtained from filtering $\sin (\tilde{\omega})$ is higher than obtained for the ODP $677 \delta^{18} \mathrm{O}$ record and results from a signal without any initial amplitude modulation. It can be inferred that if tuning is able to build in frequency modulation like that of $\sin (\tilde{\omega})$, filtering will evoke an amplitude modulation resembling eccentricity, regardless of whether the original signal is actually either frequency of amplitude modulated by eccentricity.

[20] A Monte Carlo test is designed to evaluate the efficacy with which orbital tuning generates eccentricity-like amplitude modulation. Specific results will depend upon the signal, tuning algorithm, and filtering technique that is applied, and here we attempt to adopt reasonable choices to illustrate the effect. To generate a synthetic signal, we phase randomize [Schreiber and Schmitz, 2000] the past million years of the ODP $677 \delta^{18} \mathrm{O}$ record. This gives a signal with the same spectral distribution of energy as the original $\delta^{18} \mathrm{O}$ record, but whose amplitude modulation structure is expected to have zero correlation with eccentricity. We use the depth-derived time scale discussed earlier, though results are equivalent if the tuned time scale of Shackleton et al. [1990] is instead used. 

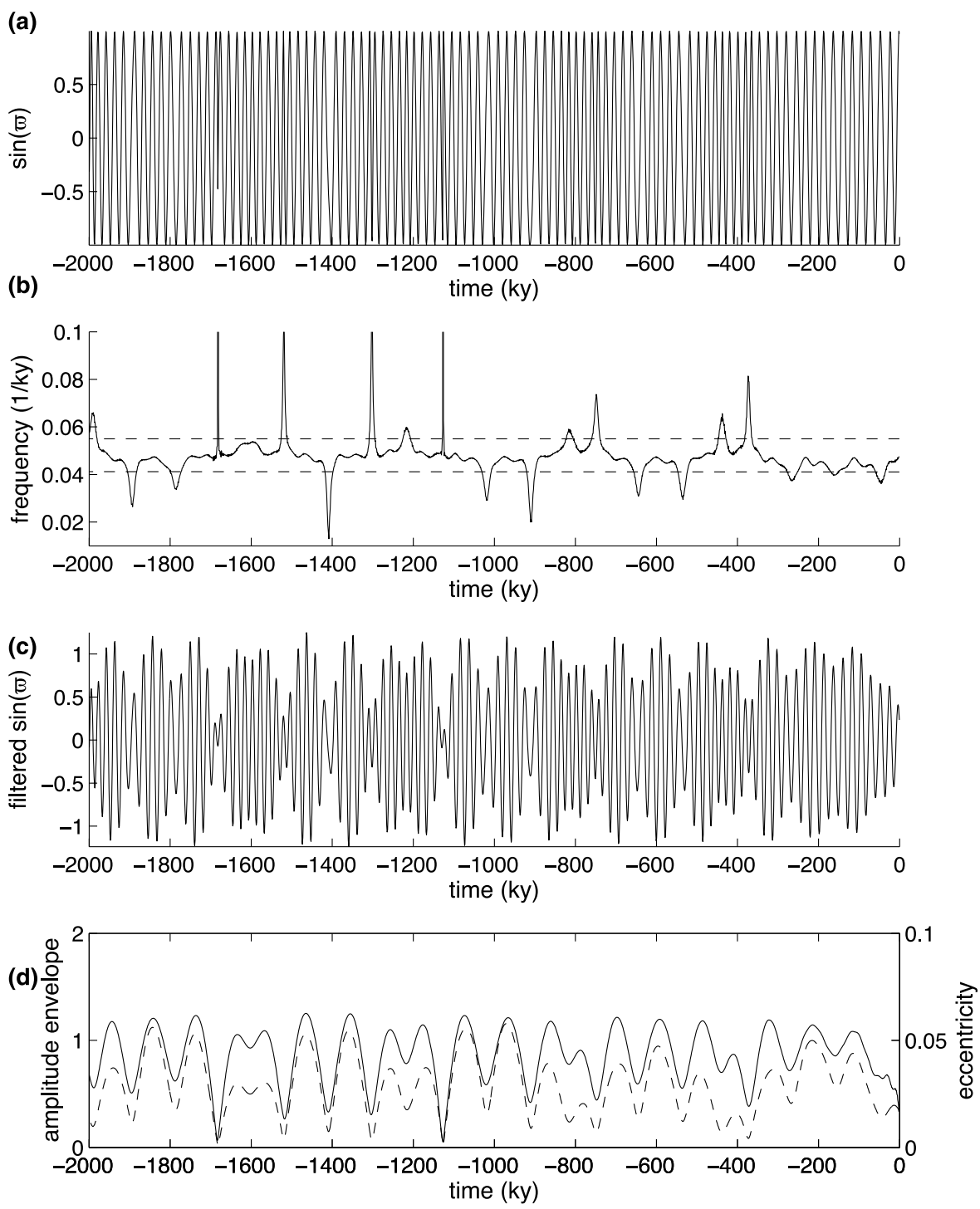

Figure 3. Conversion of frequency modulation to amplitude modulation. (a) The precession signal, sin $\tilde{\omega}$, without eccentricity amplitude modulation. (b) Time-variable frequency of the precession signal, $d \tilde{\omega} /$ $d t$. Filtering cutoff frequencies are indicated by the dashed lines at $1 / 18 \mathrm{kyr}^{-1}$ and $1 / 24 \mathrm{kyr}^{-1}$. For visual clarity, $d \tilde{\omega} / d t$ was smoothed with an $11 \mathrm{kyr}$ weighted running average prior to plotting. (c) sin $\tilde{\omega}$ after filtering. Note that the amplitude of the filtered precession signal tends to be small when the instantaneous frequency strays outside the cutoff frequencies. (d) The cross correlation between the amplitude envelope of eccentricity (dashed line) and the filtered signal (solid line) is 0.86 .

[21] We next tune the synthetic signal to climatic precession [Laskar et al., 2004]. Multiple options are available, and we select a dynamic time-warping approach [e.g., Berndt and Clifford, 1994], similar in nature to the methods described by Clark [1989] and Lisiecki and Lisiecki [2002]. The permitted time warping is regulated by a slope weighting coefficient, in this case selected to give an average standard deviation between the initial and warped time scales of 20 kyr. The cross correlation between climatic precession and the synthetic signals is initially indistinguishable from zero, and it averages 0.37 after tuning, indicating that the tuning is effective. The same Butterworth filter found to be optimal when applied to the ODP $677 \delta^{18} \mathrm{O}$ record is then applied to the synthetic signal, and the cross correlation between eccentricity and the amplitude of the filtered signal is recorded. Amplitudes are calculated using a Hilbert transform. Repeating this process a thousand times gives a mean cross correlation between the amplitude of the precession-period variability and eccentricity of 0.54 . The importance of the filtering process for evoking eccentricity modulation is highlighted by the fact that the mean cross correlation between eccentricity and the amplitude of the tuned but unfiltered signal is only 0.07 .

[22] If we apply our tuning algorithm to the actual $\delta^{18} \mathrm{O}$ record [Shackleton et al., 1990] starting from the depthderived time scale, the resulting cross correlation with 
eccentricity is 0.50 . Given a mean synthetic value of 0.54 , there is then no evidence for a significant relationship. Even the higher cross correlation of 0.61 obtained using the tuned chronology of Shackleton et al. [1990] occurs purely by chance in $30 \%$ of the random trials. (Note that Shackleton et al. [1990] tuned to an older orbital solution by Berger [1989], which depends upon the results of Laskar [1988] for the eccentricity component of the solution, whereas we have used the more recent solution by Laskar et al. [2004]. However, repeating the analysis with the older orbital solution [Berger, 1989] yields equivalent cross-correlation results out to two significant figures, indicating that the choice of orbital solution is immaterial.) Apparently, eccentricity-like amplitude modulation should ordinarily be expected when a record has been tuned to precession and then filtered.

[23] To be clear, we do not claim that Shackleton et al.'s [1990] chronology is necessarily inaccurate. Similar chronologies have been derived by tuning Mediterranean sapropels to precession [Hilgen, 1991], using sediment accumulation rates as a proxy for time [e.g., Huybers, 2007], and by radiometric dating of select events [e.g., Rohling et al., 2010], which suggests skill in Shackleton et al.'s [1990] chronology. Nor does our analysis bear upon whether precession variability and eccentricity amplitude modulation is present in ODP $677 \delta^{18} \mathrm{O}$ or other records. For instance, Shackleton et al. [1990] also conducted an analysis in the depth domain, wherein they qualitatively inferred that the amplitude modulation associated with $\sim 1 \mathrm{~m}$ length scale variations in $\delta^{18} \mathrm{O}$ is consistent with eccentricity influencing precession's amplitude. That analysis is independent of orbital tuning and, therefore, immune to the issues raised here.

\section{Conclusions}

[24] Neeman [1993] demonstrated that eccentricity-like amplitude modulation tended to result from filtering noisy records that were tuned to precession. This can be understood as the direct result of the celestial-mechanical relationship between eccentricity and the frequency of climatic precession, and from the signal-processing relationship between frequency and amplitude modulation that arises when a signal is filtered. The large excursions in the frequency of climatic precession that accompany low-eccentricity orbital configurations cause a systematic reduction in the energy passed through a filter. Filtered records containing frequency variations like those of climatic precession then have reduced amplitude during times of low eccentricity. Thus, contrary to earlier suggestions, the appearance of eccentricity-like amplitude modulation in paleoclimate records that have been tuned to precession and filtered is not diagnostic of skill in the tuned time scale. Once tuned to precession, records routinely display eccentricity-like amplitude modulation after filtering, regardless of the accuracy of the tuned time scale.

[25] A small literature is emerging regarding the statistical implications of time errors and intentional time adjustments [e.g., Thomson and Robinson, 1996; Buck and Millard, 2004; Mudelsee et al., 2009; Haam and Huybers, 2010], but this area of research remains in its infancy. Caution is warranted in drawing conclusions from records whose timing has been intentionally adjusted, particularly when the possibility of circularity exists between assumptions built into a record's chronology and the inferences derived from it. In the amplitude modulation case considered here, it was possible to substitute purely random signals for the ODP $677 \delta^{18} \mathrm{O}$ record and obtain similar results, thereby showing circularity, and analogous approaches for checking the sensitivity of results to orbital tuning should generally be possible. The failure of the amplitude modulation test underscores both the need to understand how time adjustments influence the statistical properties of a record and the need to develop general tests for the accuracy of orbitally tuned records.

\section{Appendix A}

[26] Secular theory permits for deriving a relationship between Earth's eccentricity, $e$, and the angular motion of perihelion relative to the location of vernal equinox that is referenced to a particular date, $\dot{\varpi}$ [e.g., Murray and Dermott, 1999]. A simple solution is available in the idealized case that Earth's orbit is disturbed by only one other planet. Earth can be approximated as a point mass and the disturbing planet as an elliptical ring of mass, and the solution for $e$ and $\varpi$ represented as residing in an eccentricity space having orthogonal dimensions, $e_{x}$ and $e_{y}$ (see Figure A1a).

[27] Earth's eccentricity vector, $e$, moves periodically about a point, $e_{F}$, called the forced eccentricity,

$$
\begin{gathered}
e_{x}=e_{F}+e_{f} \cos \varpi_{f} t, \\
e_{y}=e_{f} \sin \varpi_{f} t .
\end{gathered}
$$

In this example, for simplicity, the forced eccentricity is taken to lie along $e_{x}$. The periodic motion has a frequency, $\varpi_{f}$, and an amplitude, $e_{f}$, referred to as the free eccentricity.

[28] The full eccentricity vector is given by the vector sum of $e_{x}$ and $e_{y}$. The angle, $\varpi$, is given by $\tan ^{-1} \frac{e_{y}}{e_{x}}$, and its time rate of change by

$$
\dot{\varpi}=\frac{e_{x} \dot{e}_{y}-e_{y} \dot{e}_{x}}{e_{x}^{2}} \cos ^{2} \varpi .
$$

Note that the changes in eccentricity and $\varpi$ are periodic but not uniform because they are measured relative to the origin.

[29] There are two cases to consider. First, when $e_{f}$ is greater than $e_{F}, \dot{\varpi}$ increases on approaching the origin, whereas when $e_{f}$ is smaller than $e_{F}, \dot{\varpi}$ decreases. The magnitude of the effect increases with a closer approach to the origin (see Figure A1). Thus, when the eccentricity is smallest, the magnitude of the excursions in the frequency associated with precession are largest. This relationship is directly analogous to the interaction between $\tilde{\omega}$ and $e$ found in the more complete numerical simulations of Earth's orbit (Figure 2).

[30] Note that when $e_{f}$ is greater than $e_{F}$, the average value associated with $\dot{\varpi}$ equals $\dot{\varpi}_{f}$, which in this case is specified to be $1 / 100 \mathrm{kyr}^{-1}$. However, when $e_{f}$ is less than $e_{F}$, the eccentricity vector never circles around the origin and the average value associated with $\dot{\varpi}$ is zero (see Figure A1d). 

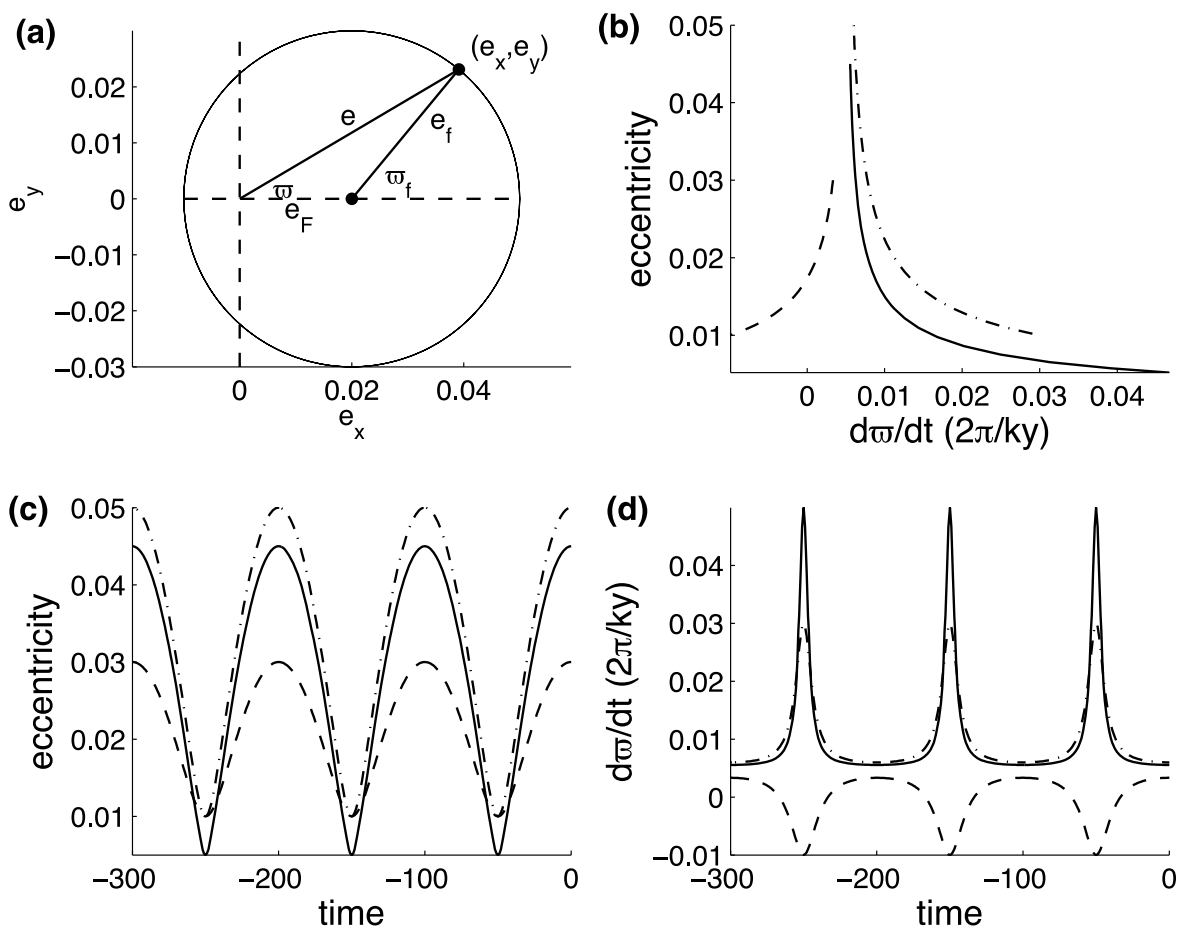

Figure A1. Eccentricity and the rate of change of the location of perihelion. (a) The eccentricity space used for illustrating the secular solution for eccentricity, $e$, and the precession angle, $\varpi$, as a function of the forced eccentricity vector, $e_{F}$, and free eccentricity vector, $e_{f}$. (b) Eccentricity versus the frequency associated with $\varpi$. (c) The time evolution of the eccentricity. (d) The frequency of $\varpi$. $e_{F}$ is specified to equal 0.02 , and $e_{f}$ to variously have values of 0.01 (dashed line), 0.025 (solid line), and 0.03 (dashed-dotted lines). Note that if $e_{f}$ is greater than $e_{F}, \dot{\varpi}$ increases when $e$ is small, but if $e_{f}$ is less than $e_{F}, \dot{\varpi}$ decreases.

For Earth, the precession of the equinoxes is more rapid than the periodic motion associated with Earth's eccentric orbit primarily because Earth's spin pole also precesses with respect to the fixed stars, but this additional effect is ignored in this simple example.

[31] Finally, note that $\varpi$ is the sum of two separate angles, $\Omega+\omega$. The longitude of the ascending node, $\Omega$, is measured as the angular distance from the fixed equinox to the timevariable location of the ascending node in the fixed plane of the ecliptic, where "fixed" refers to the geometry on a particular reference date. The argument of perihelion, $\omega$, is the angular distance from the ascending node to perihelion in the time-variable plane of the ecliptic. In the main text, we focus on the angle relevant for calculating precession's time-variable influence upon insolation, $\tilde{\omega}$, which is the angle from the time-variable position of the Northern Hemisphere spring equinox to the ascending node, $\Lambda$, plus the angle from the ascending node to perihelion, $\omega$, all measured in the time-variable plane of the ecliptic [see, e.g., Laskar et al., 1993, Figure 2]. A more complete calculation would consider the variability in $\tilde{\omega}$, as opposed to $\varpi$, but this simpler case suffices to illustrate our point.

[32] Acknowledgments. R. Sari (Hebrew University of Jerusalem) provided helpful discussion regarding orbital mechanics; C. Wunsch (MIT) and B. Bills (JPL) gave useful feedback on earlier drafts of this paper; and insightful reviews were provided by L. Lourens (Utrecht University), M. Crucifix (Université catholique de Louvain), and an anonymous reviewer.

\section{References}

Berger, A. (1976), Obliquity and precession for the last 5,000,000 years, Astron. Astrophys., 51(1), 127-135.

Berger, A. (1989), in 3rd International Conference on Paleoceanography, Cambridge, England, Terra Abstr., 1, p. 16, Blackwell Sci., Oxford, U. K.

Berggren, W., D. Kent, J. Flynn, and J. van Couvering (1985), Cenozoic geochronology, Geol. Soc. Am. Bull., 96(11), 1407-1418.

Berndt, D., and J. Clifford (1994), Using dynamic time warping to find patterns in time series, paper presented at AAAI-94 Workshop on Knowledge Discovery in Databases, Assoc. for the Adv. of Artif. Intell., Menlo Park, Calif. Bracewell, R. (2000), The Fourier Transform and its Applications, McGraw-Hill, New York.

Buck, C., and A. Millard (Eds.) (2004), Tools for Constructing Chronologies: Crossing Disciplinary Boundaries, Springer, London.

Burns, J. (1976) Elementary derivation of the perturbation equations of celestial mechanics, Am. J. Phys., 44(10), 944-949.
Clark, R. (1989), A randomization test for the comparison of ordered sequences, Math. Geol., 21(4), 429-442.

Gilbert, G. (1900), Rhythms and geologic time, Science, 11(287), 1001-1012.

Haam, E., and P. Huybers (2010), A test for the presence of covariance between time-uncertain series of data with application to the Dongge Cave speleothem and atmospheric radiocarbon records, Paleoceanography, 25, PA2209, doi:10.1029/2008PA001713. 
Hays, J., J. Imbrie, and N. Shackleton (1976), Variations in the Earth's orbit: Pacemaker of the ice ages, Science, 194, 1121-1132.

Herschel, J. (1832), On the astronomical causes which may influence geological phenomena, Trans. Geol. Soc. London, 3, 293-300, doi:10.1144/transgslb.3.2.293.

Hilgen, F. (1991), Astronomical calibration of Gauss to Matuyama sapropels in the Mediterranean and implication for the geomagnetic polarity time scale, Earth Planet. Sci. Lett., 104(2-4), 226-244.

Hilgen, F. J., L. J. Lourens, A. Berger, and M. F. Loutre (1993), Evaluation of the astronomically calibrated time-scale for the late Pliocene and earliest Pleistocene, Paleoceanography, 8 , 549-565.

Huybers, P. (2007), Glacial variability over the last two million years: An extended depthderived age model, continuous obliquity pacing, and the Pleistocene progression, Quat. Sci. Rev., 26, 37-55.

Huybers, P., and C. Wunsch (2004), A depthderived Pleistocene age-model: Uncertainty estimates, sedimentation variability, and nonlinear climate change, Paleoceanography, 19, PA1028, doi:10.1029/2002PA000857.

Imbrie, J., J. Hays, D. Martinson, A. McIntyre, A. Mix, J. Morley, N. Pisias, W. Prell, and N. Shackleton (1984), The orbital theory of Pleistocene climate: Support from a revised chronology of the marine $\delta^{18} \mathrm{O}$ record, in Milankovitch and Climate: Understanding the Response to astronomical Forcing, vol. 2, edited by A. Berger et al., pp. 269-305, D. Riedel, Dordrecht, Netherlands.

Johnson, R. (1982), Brunhes-Matuyama magnetic reversal dated at 790,000 yr B.P. by marine-astronomical correlations, Quat. Res., 17, 135-147.

Karner, D., J. Levine, B. P. Medeiros, and R. A. Muller (2002), Constructing a stacked benthic $\delta^{18} \mathrm{O}$ record, Paleoceanography, 17(3), 1030, doi:10.1029/2001PA000667.

Laskar, J. (1988), Secular evolution of the solar system over 10 million years, Astron. Astrophys., 198, 341-362.

Laskar, J., F. Joutel, and F. Boudin (1993), Orbital, precessional, and insolation quantities for the Earth from -20 myrs to $+10 \mathrm{mrs}$, Astron. Astrophys., 270, 522-533.
Laskar, J., P. Robutel, F. Joutel, M. Gastineau, A. Correia, and B. Levrard (2004), A longterm numerical solution for the insolation quantities of the Earth, Astron. Astrophys., 428(1), 261-285.

Lisiecki, L. E., and P. A. Lisiecki (2002), Application of dynamic programming to the correlation of paleoclimate records, Paleoceanography, 17(4), 1049, doi:10.1029/2001PA000733.

Lisiecki, L., and M. Raymo (2005), A PlioPleistocene stack of 57 globally distributed benthic $\delta^{18} \mathrm{O}$ records, Paleoceanography, 20 PA1003, doi:10.1029/2004PA001071.

Lourens, L. (2004), Revised tuning of Ocean Drilling Program Site 964 and KC01B (Mediterranean) and implications for the $\delta^{18} \mathrm{O}$, tephra, calcareous nannofossil, and geomagnetic reversal chronologies of the past 1.1 Myr, Paleoceanography, 19, PA3010, doi:10.1029/2003PA000997.

Lourens, L., F. Hilgen, I. Raffi, and C. VergnaudGrazzini (1996), Early Pleistocene chronology of the vrica section (Calabria, Italy), Paleoceanography, 11(6), 797-812, doi:10.1029/ 96PA02691.

Lourens, L., F. Hilgen, N. Shackleton, J. Laskar, and D. Wilson (2004), The neogene period, in A Geologic Time Scale 2004, chap. 21, pp. 409-440, Cambridge Univ. Press, Cambridge, U. K.

McGee, W. (1892), Comparative chronology, Am. Anthropol., pp. 327-344.

Mudelsee, M., D. Scholz, R. Röthlisberger, D. Fleitmann, A. Mangini, and E. Wolff (2009), Climate spectrum estimation in the presence of timescale errors, Nonlinear Processes Geophys., 16, 43-56.

Muller, R., and G. MacDonald (2000), Ice Ages and Astronomical Causes, Springer, London.

Murray, C., and S. Dermott (1999), Solar System Dynamics, Cambridge Univ. Press, Cambridge, U. K.

Neeman, B. (1993), Orbital tuning of paleoclimate records: A reassessment, $L B N L-39572$, Lawrence Berkeley Lab., Berleley, Calif.

Olver, F. W. (1962), Tables for Bessel Functions of Moderate or Larger Orders, H. M. Station. Off., London.

Paillard, D. (2001), Glacial cycles: Towards a new paradigm, Rev. Geophys., 39(3), 325-346.
Quinn, T., S. Tremaine, and M. Duncan (1991), A three million year integration of the Earth's orbit, Astron. J., 101, 2287-2305.

Rohling, E., K. Braun, K. Grant, M. Kucera, A. Roberts, M. Siddall, and G. Trommer (2010), Comparison between Holocene and Marine Isotope Stage-11 sea-level histories, Earth Planet. Sci. Lett., 291, 97-105.

Rubincam, D. (1994), Insolation in terms of Earth's orbital parameters, Theor. Appl. Climatol., 48, 195-202.

Ruddiman, W. F., M. Raymo, D. Martinson, B. Clement, and J. Backman (1989), Pleistocene evolution: Northern Hemisphere ice sheets and the North Atlantic Ocean, Paleoceanography, 4, 353-412, doi:10.1029/PA004i004p00353.

Schreiber, T., and A. Schmitz (2000), Surrogate time series, Physica D, 142, 346-382.

Shackleton, N. J., A. Berger, and W. R. Peltier (1990), An alternative astronomical calibration of the lower Pleistocene timescale based on ODP site 677, Trans. R. Soc. Edinburgh, 81, 251-261.

Shackleton, N. J., T. K. Hagelberg, and S. J. Crowhurst (1995), Evaluating the success of astronomical tuning: Pitfalls of using coherence as a criterion for assessing pre-Pleistocene timescales, Paleoceanography, 10, 693-697, doi:10.1029/95PA01454.

Thomson, P. J., and P. M. Robinson (1996), Estimation of second-order properties from jittered time series, Ann. Inst. Stat. Math. 48, 29-48.

Tiedemann, R., M. Sarnthein, and N. J. Shackleton (1994), Astronomic timescale for the Pliocene Atlantic $\delta^{18} \mathrm{O}$ and dust flux records of ODP site 659, Paleoceanography, 9, 619-638, doi:10.1029/94PA00208.

Williams, J. (1994), Contributions to the Earth's obliquity rate, precession, and nutation, Astron. J., 108, 711-724.

O. Aharonson, Division of Geological and Planetary Sciences, California Institute of Technology, MC 150-21, Pasadena, CA 91125 , USA.

P. Huybers, Department of Earth and Planetary Sciences, Harvard University, Museum Building, Rm. 405, Cambridge, MA 02138 , USA. (phuybers@fas.harvard.edu) 